\title{
Morbidity and mortality from COVID-19 post- vaccination breakthrough infections in association with vaccines and the emergence of variants in Bahrain
}

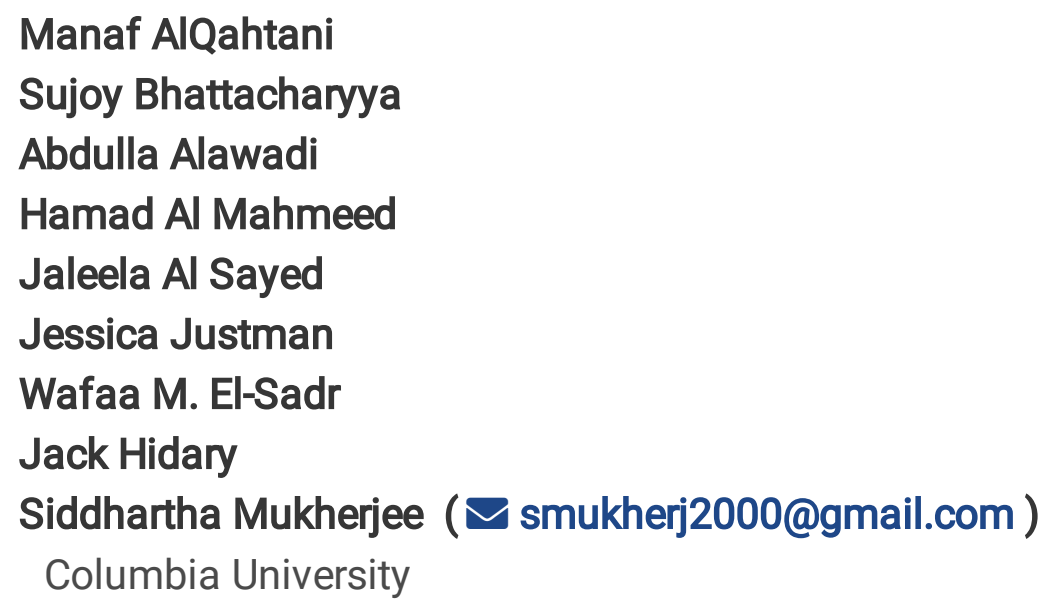

Biological Sciences - Article

Keywords: SARS-CoV-2, COVID-19, variants, vaccines, Delta variant

Posted Date: August 24th, 2021

DOl: https://doi.org/10.21203/rs.3.rs-828021/v1

License: (c) (i) This work is licensed under a Creative Commons Attribution 4.0 International License. Read Full License 
TITLE: Morbidity and mortality from COVID-19 post-vaccination breakthrough infections in association with various COVID-19 vaccines and the emergence of variants in the Kingdom of Bahrain

Authors: Manaf AlQahtani ${ }^{1,2}$, Sujoy Bhattacharyya', Abdulla Alawadi,2, Hamad Al Mahmeed ${ }^{1,2}$, Jaleela Al Sayed ${ }^{1,2}$, Jessica Justman ${ }^{4}$,Wafaa M. El-Sadr, Jack Hidary ${ }^{5}$ and Siddhartha Mukherjee ${ }^{6}$

\section{Bahrain Defense Force Hospital}

2. National Task Force for Combating COVID-19, Kingdom of Bahrain

3.Department of Economics, Columbia University

4. ICAP at Columbia University, Mailman School of Public Health, New York

5. Alphabet, Mountain View

6. Division of Oncology, Department of Medicine, Columbia University, New York

ABSTRACT: The emergence of new SARS-CoV-2 variants across the world has raised concerns about the effectiveness of available COVID-19 vaccines that were designed against the original Wuhan (wild type) variant. Critical questions have arisen regarding: (a) the effectiveness of various vaccines in preventing infection, symptomatic disease, hospitalization, intensive care unit (ICU) admission and death and (b) the magnitude and clinical consequences of post-vaccination infections in the context of emerging variants, especially the Delta variant of SARS-Cov2. While "real world" experiences with various vaccines have been reported, few have examined comparative effectiveness of various vaccines in one population, as new SARS-CoV-2 variants have emerged. Here we present an analysis of COVID-19 related outcomes from Bahrain, a country with a total population of 1.501 million, where four vaccines were deployed (total vaccinated =1,003,960), including Astra-Zeneca (AZ/Covishield), Pfizer/BioNtech, Sinopharm and Sputnik V. By analyzing individual histories of vaccinated versus unvaccinated cases, we provide a granular description of the effectiveness of the four vaccines, disease burden in unvaccinated versus vaccinated individuals over time, and the risk of four outcomes (infections, hospitalizations, ICU admissions and deaths) due to breakthrough infections among vaccinated individuals. We conclude that the four vaccines were effective in reducing all four outcomes in vaccinated compared to unvaccinated individuals, prior to, and during the period when the Delta variant became dominant in the country (May 2021 to the present). However, after censoring early vaccine recipients of Sinopharm vaccine, compared to Pfizer/BionTech recipients, individuals vaccinated with Sinopharm had a higher risk of post-vaccination infections, hospitalizations, ICU admissions and deaths, especially in those $>50$ years old. Our overall findings support the value of vaccination in preventing COVID-19 related events even with the advent of the Delta variant. These data support the urgent need to expand vaccination access around the world, and may serve to guide the choice of vaccines in the context of the Delta variant.

Vaccination offers a critical tool for the control of the COVID-19 pandemic. To date, multiple vaccines have been developed around the world that utilize different technologies. ${ }^{1-5}$ Clinical trials have demonstrated variable levels of protection against symptomatic COVID-19, the primary outcome for many of these studies. However, these clinical trials were conducted at different times during the pandemic, in different countries and with varying prevailing SARS-CoV-2 variants. There have been no direct head-to-head comparisons of efficacy across vaccines, limited comparisons of effectiveness in a 
real-world context as new variants predominate over time, and limited analysis of breakthrough, post vaccination infections comparing multiple vaccines in one population. ${ }^{6}$

The Government of Bahrain's decision to deploy four vaccines provided an unparalleled opportunity to examine and directly compare the effectiveness of different vaccines in terms of prevention of COVID19 cases, hospitalizations, ICU admissions and deaths over time in a large population as new variants were emerging.

\section{Vaccination Uptake:}

Between December 9th 2020 and July 17th 2021, 569,054 individuals were vaccinated with Sinopharm, 184,526 with Sputnik, 73,765 with AstraZeneca (AZ/Covishield) and 169,058 with Pfizer/BioNtech vaccines, while 245,876 were used as the unvaccinated case-control cohort (Fig 1). The unvaccinated included travelers, asymptomatic and symptomatic contacts of those who had tested positive for SARSCOV2, patients who had symptoms consistent with SARS-Cov2 infection, individuals undergoing operative and elective medical procedures, and individuals undergoing random tests in areas of high population density. We censored in our analyses the population between 12-17 years of age in the Pfizer/BioNtech group.

We determined the date of achievement of presumptive protection (PP), defined as the date corresponding to 14 days after receipt of the second dose of any of the four vaccines, as each involved a two-dose regimen. As noted in Figure 1C, the Sinopharm vaccine was the first and most frequently used vaccine in Bahrain (total number of recipients: 569,054; date of PP of first vaccine recipients: December 9th, 2020; date of 50\% rollout: April, 24, 2021). Pfizer/BioNtech vaccine rolled out next (total number of recipients 169,058; date of PP of first vaccine recipients: Jan 30, 2021, date of 50\% rollout: June 14, 2021). AZ/Covishield (total number of recipients: 73,765; date of PP of first vaccine recipients: March 13, 2021, date of 50\% rollout: April 24, 2021), followed by Sputnik V (total number of recipients: 184,526; date of PP of first vaccine recipients: March 4, 2021, date of 50\% rollout: May 30, 2021).

\section{Characteristics of vaccinated individuals}

The median age of individuals vaccinated was similar across the four vaccines utilized: Sinopharm: 38 years, AZ/Covishield: 39 years, Sputnik V: 35 years, Pfizer BioNtech: 36 years (vaccinated individuals 12-18 years were censored). There were, however, differences in the sex distribution. While with Pfizer/BioNtech and Sinopharm vaccines, there was a similar distribution ( $63.4 \%$ males $/ 36.6 \%$ females and $60.9 \%$ males $/ 39.1 \%$ females respectively), men were more likely to have received the Sputnik V and AZ/Covishield vaccines with $73.4 \%$ male $/ 26.6 \%$ female and $81.9 \%$ males $/ 18.1 \%$ females, respectively.

\section{SARS-Cov2 PCR testing}

Beginning in February, 2020, the COVID-19 task force of Bahrain began testing all arriving travelers into the country, suspected cases, symptomatic individuals, asymptomatic contacts (including family members) of those who had tested positive, and all hospitalized and critically ill patients suspected of being infected by SARS-Cov2. Infection monitoring was substantially expanded after Dec 1, 2020 and reached a peak of an average of 15,000 tests per day by February 2021. Testing was performed regardless of vaccination status. 


\section{Sequencing of SARS-CoV-2 isolates}

Beginning in June 2020, variant sequencing was initiated on isolates from a few COVID-19 cases including travelers, symptomatic cases and critically ill patients and post vaccination cases. Variant sequencing was greatly increased in February 2021 and has continued until July 29, 2021, by which time a total of 7,807 isolates have been sequenced. The results of variant testing, starting in February 2021, are shown in Figure 2. Variants were initially dominated by the Beta/B.1.351 variant (between March and April 2021), followed by a rise in Delta/B.1.617.2 and Kappa/ B.1.617.1 variants (between April through June, 2021). The representation of the Delta/B.1.617.2 variant continued to rise from April through July, 2021. We measured the fraction of the Delta variant among all PCR positive cases, and found that fifty percent of the cases were of the Delta variant by May 12021 (Fig 2b). In subsequent analysis, we use May 1 (red line, Fig 2b) as the cutoff date for the dominance of the Delta variant.

\section{COVID-19 outcomes}

There were a total of 180,840 PCR positive COVID-19 cases identified among all individuals, with 134,728 among those 18 years and older. There were 13,105 hospitalizations, including 1,636 ICU admissions, and a total of 1,030 deaths. We assessed COVID-19 hospitalizations, ICU admissions and deaths by vaccination status. We further examined the data by age, for those $>50$ versus $\leq 50$ years of age, taking into account the known increase in risk of COVID-19 related morbidity and mortality among those $>50$ years of age. ${ }^{7,8}$ As noted above, we restricted our analyses to include only individuals $\geq 18$ years of age among recipients of the Pfizer/BioNtech vaccine. In addition, in order to limit multiple comparisons, we did paired testing among five groups: Unvaccinated vs Sinopharm, Unvaccinated vs Pfizer/BioNtech, Unvaccinated vs AZ/Covishield, Unvaccinated vs Sputnik V. Using pairwise comparisons between unvaccinated individuals ( $>50$ and $\leq 50$ years), we found significantly higher $\%$ of hospitalization, ICU admissions and deaths in the unvaccinated group compared to the vaccinated group for all four vaccines (Tables 1). For instance, the percent of deaths among all COVID-19 cases in unvaccinated people $>50$ years of age was 3.8-fold higher compared to the Sinopharm, and approximately 7.5-fold higher than the Sputnik V vaccine recipients. There were no deaths reported in the AZ/Covishield vaccinated group. In patients $\leq 50$ years, the percent of death among all COVID-19 cases in unvaccinated people were 8.1-fold higher compared to Sinopharm vaccine recipients. There were no deaths reported in this age cohort in Sputnik V and Pfizer/BioNtech vaccine recipients, and 1 death among the AZ/Covishield vaccine recipients (Table 1).

Despite the overall effectiveness of all four vaccines in decreasing risk of COVID-19 related hospitalizations, intensive care unit admissions and deaths when compared to unvaccinated individuals, our initial analysis showed that there was a higher risk of COVID-19 infection and clinical escalation among recipients of the Sinopharm vaccine compared to other vaccine recipients. For instance, the percentage of deaths among all PCR positive post-vaccination COVID-19 cases among recipients of the Sinopharm vaccine was $0.46 \%$ (i.e, 112 deaths) versus $0.15 \%$ for Pfizer/BioNtech (i.e., 3 deaths) and $0.03 \%$ for AZ/Covishield (1 death) (Table 1). This trend was consistent for all COVID-19 events, i.e., infection, hospitalization, ICU admission and death (Tables 1). 
To further examine this issue, we restricted the comparison of Pfizer/BioNtech versus Sinopharm vaccines (the two vaccines are demographically comparable in sex and age distribution and were also temporally deployed within a month of one another). We censored early recipients of Sinopharm (any vaccinated individuals who had achieved presumptive protection on January 30, 2021 or before that date; this date was chosen because it corresponds to the first individuals achieving presumptive protection after Pfizer/BioNtech vaccination). Furthermore, to avoid multiple comparisons among five groups, we used the data for the Pfizer/BioNtech vaccine as the comparator. We found that the Pfizer/BioNtech vaccine was associated with statistically significantly lower rates of post-vaccination PCR positive COVID-19 infections, hospitalizations, ICU admissions and deaths compared to the Sinopharm vaccine among those $>50$ years of age. This was also noted for those $\leq 50$ years of age, although the difference in the death rates in this cohort was not statistically significant; this may have been due to low numbers of events (Table 2).

\section{Timeline of COVID-19 Events}

To determine how rates of COVID-19 infection, hospitalization, ICU admission and death occurred over time, we constructed a time series for unvaccinated individuals and recipients of the four vaccines. Rates were calculated by dividing the number of cases (positive tests, hospitalization, ICU admissions, or deaths) by the accumulated total number of people vaccinated (for each vaccine type -- i.e., events per person at risk per day). This calculation was performed for each day to produce a time series. Unvaccinated rates were obtained by dividing the daily number of cases by the total number of unvaccinated people in the population.

As shown in Figure 3, we found a peak in all four clinical outcomes in the May and June 2021 period, which corresponds to the rise in the Delta variant in the country. As above, all four vaccines decreased the rates of infection, hospitalization, ICU admission and death (Figure 4). The rates of ICU admission and deaths, however, were higher among the Sinopharm vaccinees compared to other vaccine recipients. To formally compare the morbidity and mortality in post-vaccination infections for the vaccines during this period of time, and avoid multiple cross-comparisons, we restricted our comparison to examining the rates of post-vaccination infections causing hospitalizations, ICU admissions or death for the Pfizer/BioNtech and Sinopharm vaccine recipients. (Figure 4). We found that the recipients of the Sinopharm vaccine had significantly higher rates of hospitalization, ICU admission and death compared to recipients of the Pfizer/BioNtech vaccine, especially among those 50 years of age or older (Figure 4). We also constructed a time series of post-vaccination infections, hospitalizations, ICU admissions and deaths disaggregated by age of the vaccine recipient (50 years or younger and older than 50 years) (Figure S1). Consistent with our prior data, hospitalizations, ICU admissions and deaths were also highest in the older Sinopharm recipients, with a peak during the May/June 2021 period, compared to younger recipients (age $<50)$ and recipients of the other three vaccines.

\section{Variant sequencing and mortality}

Sequencing for variants was performed on all the people who had died with SARS-Cov2 infection after Jan 1 2021. As shown in Fig 5 below, prior to May 2021, deaths were dominated by unvaccinated individuals infected predominantly by the Alpha variant. After May 2021, deaths began to rise in the vaccinated population, and both unvaccinated and vaccinated deaths were dominated by the Delta variant (e.g., in May 2021, there were 135 deaths in the unvaccinated cohort, 117 of which were positive 
for the Delta variant; in the vaccinated group, there were 53 deaths, with 45 that tested positive for the Delta variant. Sequencing analysis has not been completed for July (Figure 5).

\section{Discussion:}

The ambitious vaccination program in Bahrain, combined with detailed individual case monitoring, allowed us an unprecedented opportunity to examine the real-world effectiveness of the four vaccines included in the program overall and since the advent of the Delta variant. The uptake of the Sinopharm vaccine was nearly 5-fold higher compared to the Pfizer/BioNtech, AZ/Covishield and Sputnik V vaccines as it was deployed first, and because Bahrain participated in several trials in collaboration with Sinopharm. Nonetheless, the median age of vaccinations was similar (Pfizer/BioNtech vaccine was offered to those between 12-16 years of age, but these data were censored from analysis). We also censored the early recipients of the Sinopharm vaccine - i.e., individuals who had been fully immunized on or before January 30, 2021.

Our findings indicate that all four vaccines decreased the risk of SARS-CoV-2 infections, hospitalizations, ICU admissions and deaths among vaccine recipients when compared to unvaccinated individuals. However, individuals who received the Sinopharm experienced a higher rate of infection, hospitalization, ICU admission and deaths compared to those who received the Pfizer/BioNtech vaccine. Outcomes for the Sputnik V vaccinated individuals were intermediate. Targeted sequencing of virus isolates from individuals who died from COVID-19 showed that deaths from January through April 2021 were dominated by the Alpha variant, as the most dominant variant in the country at that time. From May 2021 onwards, the fraction of all infections caused by the Delta variant rose from 30 to 90 percent by July 2021. Death rates associated with the emergence of the Delta variant also rose in May, 2021 as anticipated based on increase in the number of cases, with this variant identified in 86 percent among the deaths noted for unvaccinated and 84 percent among the deaths in the vaccinated group. In June, 2021 all deaths in both groups were caused by the Delta variant, the dominant variant in the country.

Our study has some limitations. The limited information on socioeconomic and clinical characteristics of the individuals included in the analyses, hindered the ability to take into account any adverse selection that might have occurred due to geographic access and clinical characteristics and their effects on the uptake of different vaccines. Similarly, we recognize that evolution of the adoption of pandemic protective measures changed over time, which may have influenced risk for SARS-CoV-2 infection. In particular, we note that Sinopharm was the only vaccine used early in the pandemic when knowledge of diagnosis and management of cases of COVID-19 was evolving; however, we censored early recipients of the Sinopharm vaccine to mitigate any bias caused by this early cohort of recipients.

Since the Sinopharm vaccine was deployed at an approximately three-fold higher rate compared to the Pfizer/BioNtech vaccine, we cannot rule out a skew in the data due to oversampling of individuals who received the Sinopharm vaccine and experienced infections, hospitalizations, ICU admissions and deaths. Additionally, it is important to note that testing efforts included close contact of cases of COVID-19, which may have selected a group at higher risk for infection. On the other hand, given the highly significant differences noted in post-vaccination infection rates with clinical consequences in the Sinopharm compared to the Pfizer/BioNtech vaccine recipients, especially among those older than 50 
years of age in terms of hospitalizations and deaths, it is unlikely that these confounding factors could explain the difference in outcomes between the two vaccines.

We conclude that COVID-19 vaccination is an effective strategy at mitigating the risk for SARS-CoV-2 and its consequences, especially among individuals older than 50 years of age. However, in a pairwise comparison, the performance of the Pfizer/BioNtech vaccine was superior to the Sinopharm vaccine, especially in older individuals and in the context of the emergence of the Delta variant.
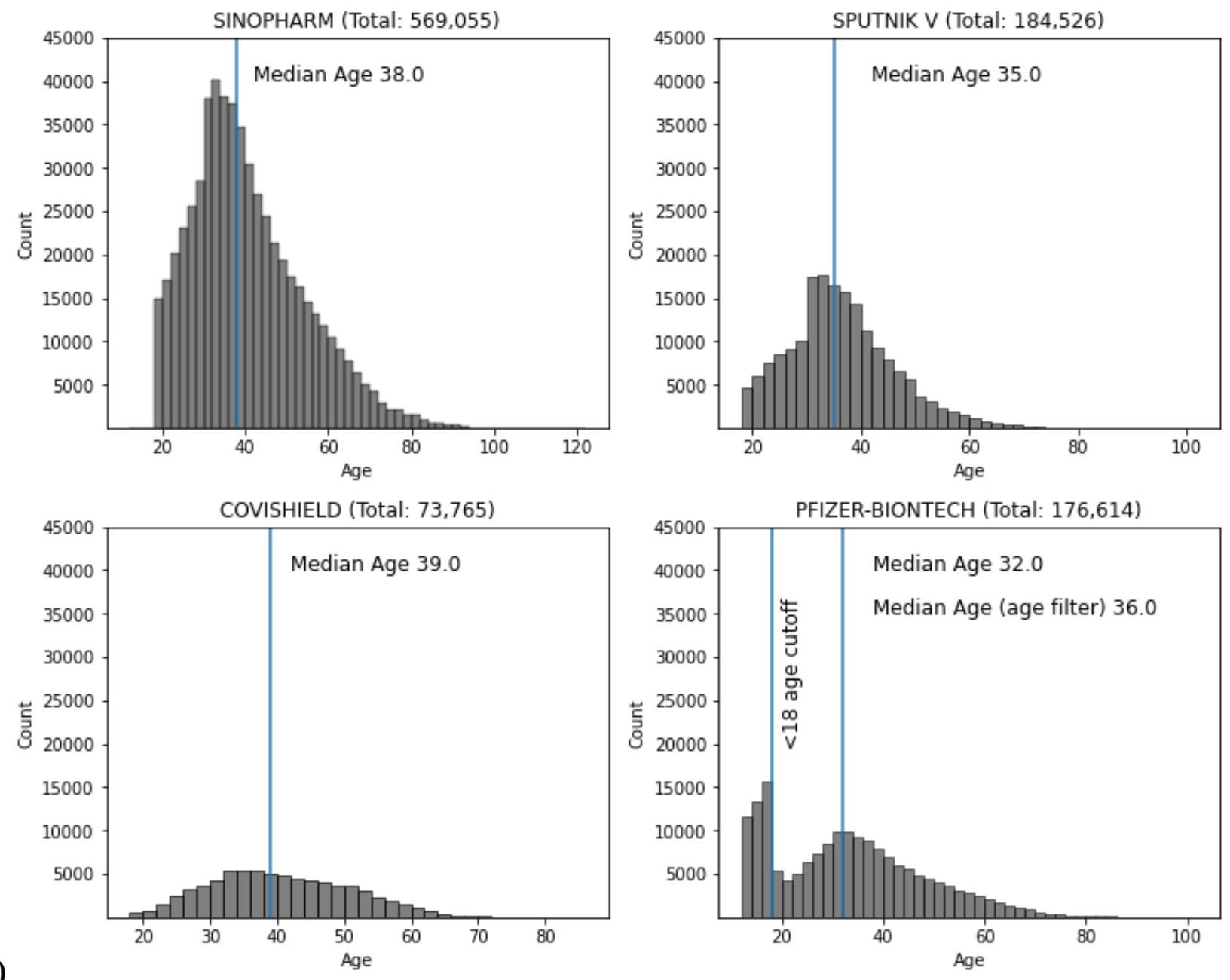

(A)

(B) 

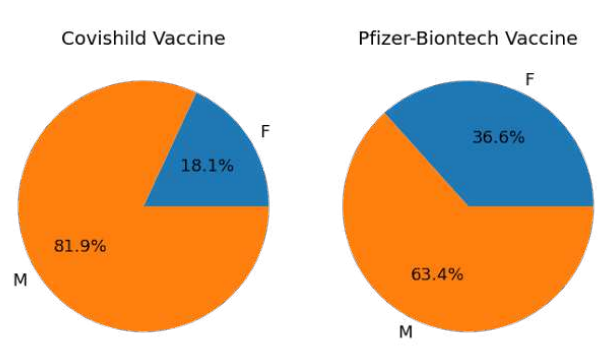

Per Vaccine Gender Demographics
Sinopharm Vaccine
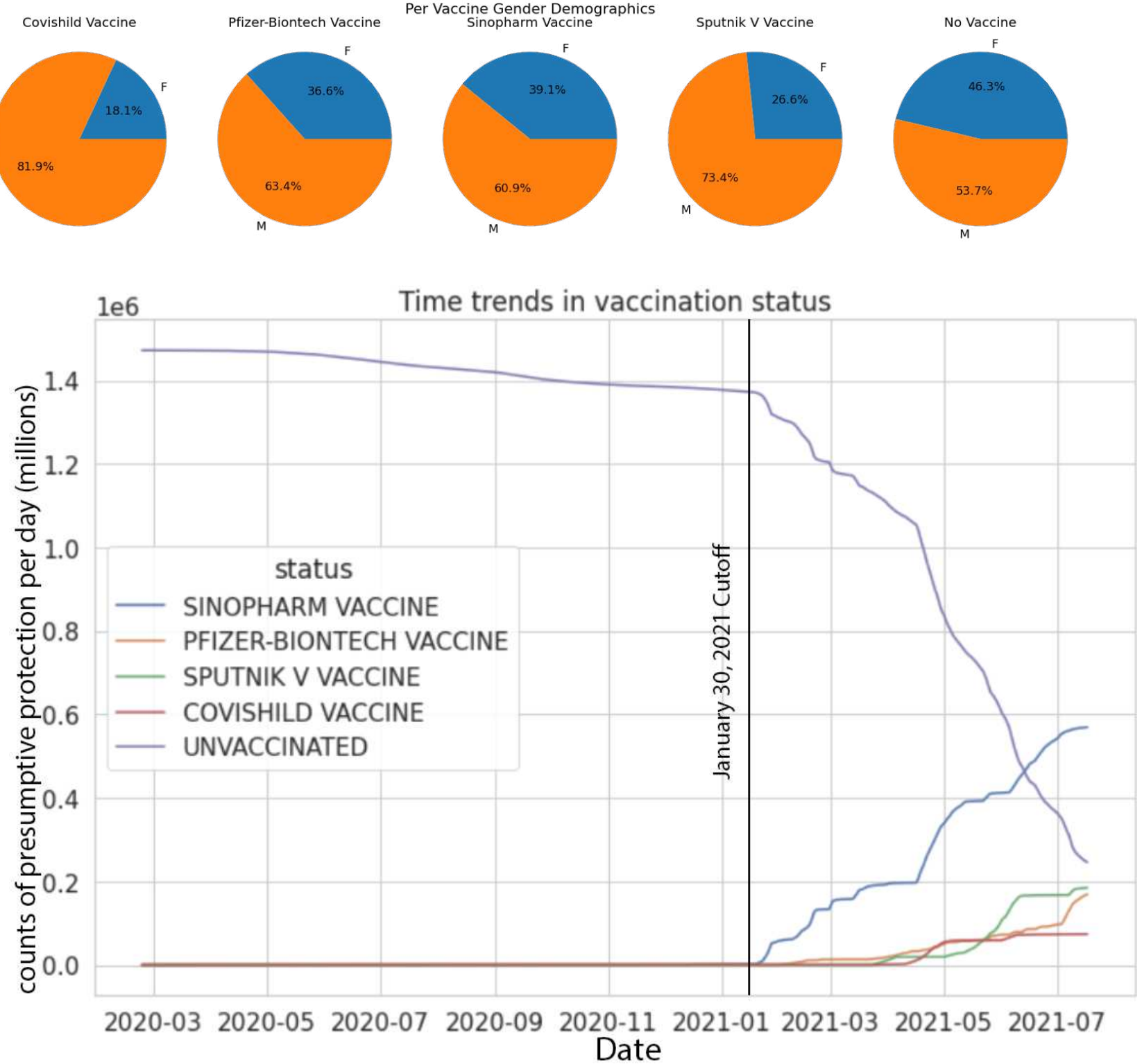

(C)

Figure 1. (A) Age distributions of the four different vaccines administered in the population. PfizerBioNTech was the only vaccine given to ages $12-18<18$ years of age, and those data were excluded 
from the analysis (B) Age and gender demographics for all vaccines. (C) Visualization of the vaccine rollout. The curves display the counts of individuals that have or do not have full vaccination status, or presumptive protection (i.e,. 14 days after the booster dose) for each of the four vaccines on each day. The number of individuals without presumptive protection (PP) on each day drops as vaccination is rolled out. January 30th is the date that the first Pfizer recipients achieved presumptive protection (i.e.,14 days after their second Pfizer vaccination dose) and is therefore used as a cutoff to censor any early recipients of Sinopharm.
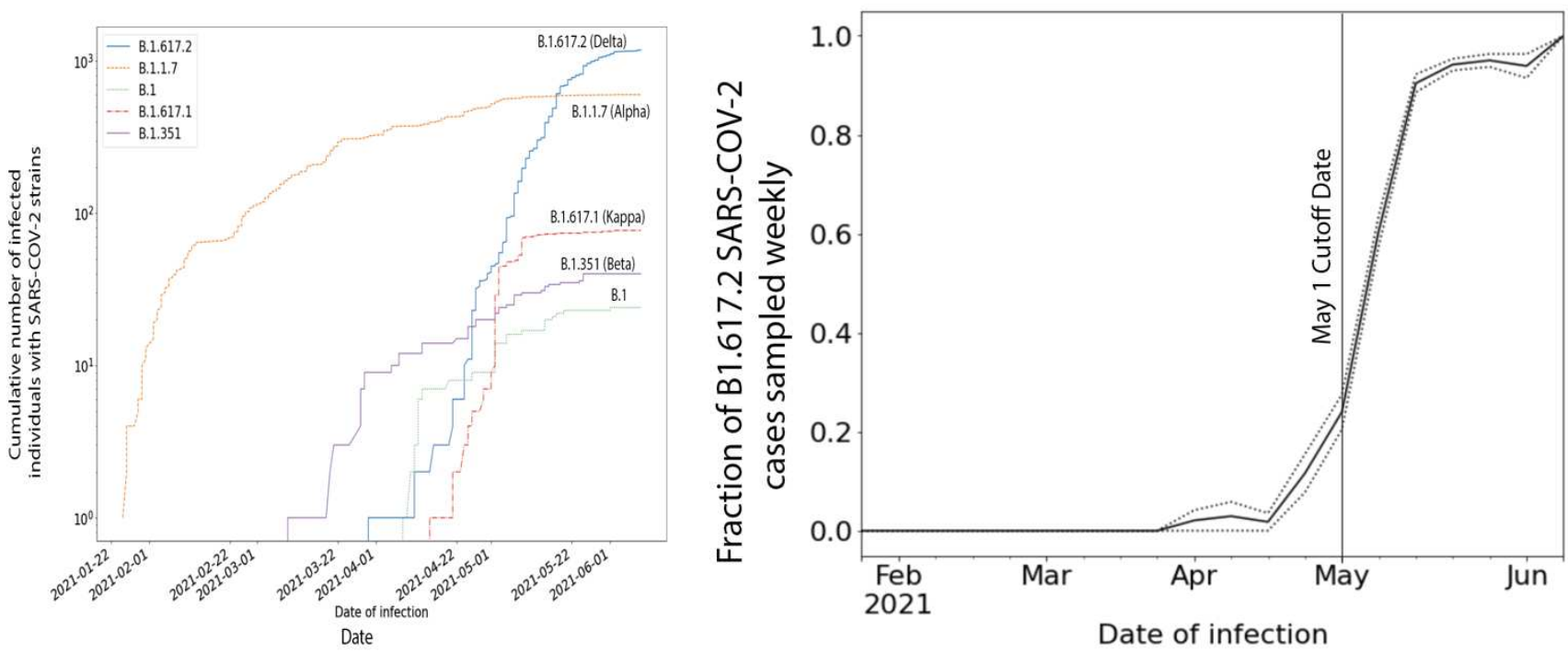

(a)

(b)

Figure 2: (a) Cumulative counts of individuals infected with various strains of SARS-Cov2 in Bahrain. (b) Fraction of infections of Delta/B.1.617.2 (over all other variants detected) with +- one s.e. of the total fraction. Total counts of B.1.617.2 exceed other detections (note log scale). May 1 (red line) was selected as a cutoff date of when B.1.617.2 began to rise as the dominant strain in the population.

Table 1. Hospitalization (\%) of SARS-COV-2 PCR positive cases (18+Ages) 


\begin{tabular}{|c|c|c|c|c|c|c|c|c|c|c|}
\hline & \multirow[b]{2}{*}{ Vaccine Type } & \multicolumn{3}{|c|}{$\begin{array}{c}\text { Total Rate } \\
\text { (Hospitalizations) }\end{array}$} & \multicolumn{3}{|c|}{ > 50 Rate (Hospitalizations) } & \multicolumn{3}{|c|}{$\begin{array}{r}<50 \text { Rate } \\
\text { (Hospitalizations) }\end{array}$} \\
\hline & & Rate (Total) & $\begin{array}{r}\text { Pai } \\
\text { Comp }\end{array}$ & $\begin{array}{l}\text { wise } \\
\text { risons }\end{array}$ & Rate (Total & $\begin{array}{l}\text { Pairv } \\
\text { Compa }\end{array}$ & $\begin{array}{l}\text { ise } \\
\text { isons }\end{array}$ & Rate (Total & $\begin{array}{l}\text { Pair } \\
\text { Comp }\end{array}$ & $\begin{array}{l}\text { wise } \\
\text { arisons }\end{array}$ \\
\hline 1 & Unvaccinated & $13.22(8602)$ & $12 \frac{2}{T}$ & 34 & $31.83(3583)$ & 56 & 78 & $9.33(5019)$ & 910 & 1112 \\
\hline 2 & Sinopharm & $6.94(1683)$ & 1 & & $16.10(1081)$ & 1 & & $3.43(602)$ & & \\
\hline 3 & Sputnik V & $2.24(77)$ & 1 & & $11.87(45)$ & 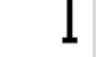 & & $1.05(32)$ & & \\
\hline 4 & Pfizer-BioNtech & $1.99(40)$ & & 1 & $4.85(21)$ & & 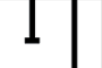 & $1.21(19)$ & & \\
\hline 5 & Covishield & $1.52(45)$ & & 1 & $4.19(24)$ & & 1 & $0.88(21)$ & & 1 \\
\hline
\end{tabular}

Pairwise Comparisons (All p-values < 0.001): 1. OR: 2.04 ; 2. OR: 6.64 ;3. OR: 7.50;4. OR: 9.84 ; 5. OR: 2.43 ;6. OR: 3.46; 7. OR: 9.16; 8. OR: 10.68; 9. OR: 2.89;10. OR: 9.72; 11. OR: 8.43 ;12. OR 11.56.

ICU admission (\%) of all SARS-Cov2 positive cases (18+ Ages)

\begin{tabular}{|c|c|c|c|c|c|c|c|c|c|c|}
\hline \multirow{2}{*}{\multicolumn{2}{|c|}{ Vaccine Type }} & \multicolumn{3}{|c|}{$\begin{array}{c}\text { Total Rate (ICU } \\
\text { Admissions) }\end{array}$} & \multicolumn{3}{|c|}{$\begin{array}{r}>50 \text { Rate (ICU } \\
\text { Admissions) }\end{array}$} & \multicolumn{3}{|c|}{$\begin{array}{r}<50 \text { Rate (ICU } \\
\text { Admissions) }\end{array}$} \\
\hline & & \multirow{2}{*}{\begin{tabular}{|r|} 
Rate (Total) \\
$2.01(1309)$
\end{tabular}} & \multicolumn{2}{|c|}{$\begin{array}{c}\text { Pairwise } \\
\text { Comparisons }\end{array}$} & \multirow{2}{*}{$\begin{array}{r}\text { Rate (Total } \\
7.6(855)\end{array}$} & \multicolumn{2}{|c|}{$\begin{array}{c}\text { Pairwise } \\
\text { Comparisons }\end{array}$} & \multirow{2}{*}{$\begin{array}{c}\text { Rate (Total } \\
0.84(454)\end{array}$} & \multicolumn{2}{|c|}{$\begin{array}{c}\text { Pairwise } \\
\text { Comparisons }\end{array}$} \\
\hline 1 & Unvaccinated & & 12 & $\begin{array}{ll}3 & 4 \\
T\end{array}$ & & 56 & 78 & & 910 & 1112 \\
\hline 2 & Sinopharm & $0.57(138)$ & 1 & & $1.7(114)$ & & & $0.14(24)$ & & \\
\hline 3 & Sputnik V & $0(0)$ & 1 & & $0(0)$ & 1 & & $0(0)$ & & \\
\hline 4 & Pfizer-BioNtech & $0.05(1)$ & & 1 & $0.23(1)$ & & 1 & $0(0)$ & & \\
\hline 5 & Covishield & $0.1(3)$ & & 1 & $0.17(1)$ & & & $0.08(2)$ & & \\
\hline
\end{tabular}

Pairwise Comparisons (All p-values < 0.001): 1. OR: 3.59 ; 2. OR: inf ;3. OR: 41.2; 4. OR: 20.20; 5. OR: 4.76; 6. OR: inf (no ICU); 7. OR: 35.51; 8. OR: 47.03; 9. OR: 6.21;10. OR: inf (no ICU) ; 11. OR: inf (no ICU);12. OR 10.12.

Deaths (\%) of all SARS-Cov2 positive cases (18+Ages)

\begin{tabular}{|c|c|c|c|c|c|c|c|c|c|c|}
\hline \multirow[b]{3}{*}{1} & \multirow[b]{3}{*}{ Unvaccinated } & \multicolumn{3}{|c|}{ Total Rate (Deaths) } & \multicolumn{3}{|c|}{$>50$ Rate (Deaths) } & \multicolumn{3}{|c|}{$<50$ Rate (Deaths) } \\
\hline & & \multirow{2}{*}{\begin{tabular}{|r|} 
Rate (Total) \\
$1.32(857)$
\end{tabular}} & \multicolumn{2}{|c|}{$\begin{array}{c}\text { Pairwise } \\
\text { Comparisons }\end{array}$} & \multirow{2}{*}{\begin{tabular}{|l|} 
Rate (Total \\
$5.64(635)$ \\
\end{tabular}} & \multicolumn{2}{|c|}{$\begin{array}{c}\text { Pairwise } \\
\text { Comparisons }\end{array}$} & \multirow{2}{*}{$\begin{array}{c}\text { Rate (Total } \\
0.41(222)\end{array}$} & \multicolumn{2}{|c|}{$\begin{array}{c}\text { Pairwise } \\
\text { Comparisons }\end{array}$} \\
\hline & & & 12 & 34 & & 56 & 78 & & 910 & 1112 \\
\hline 2 & Sinopharm & $0.46(112)$ & 1 & & $1.53(103)$ & & & $0.05(9)$ & & \\
\hline 3 & Sputnik V & $0.09(3)$ & 1 & & $0.79(3)$ & 1 & & $0(0)$ & & \\
\hline 4 & Pfizer-BioNtech & $0.15(3)$ & & 1 & $0.69(3)$ & & 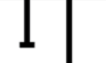 & $0(0)$ & & 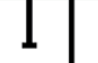 \\
\hline 5 & Covishield & $0.03(1)$ & & 1 & $0(0)$ & & & $0.04(1)$ & & \\
\hline
\end{tabular}

Pairwise Comparisons (All p-values < 0.001): 1. OR: 2.88; 2. OR: 15.27 ;3. OR: 8.92; 4. OR: 39.41; 5. OR: 3.83; 6. OR: 7.49; 7. OR: 8.57; 8. OR: inf (no deaths); 9. OR: 8.07;10. OR: inf (no deaths) ; 11. OR: inf (no deaths); 12. OR 39.41.

Table 1: Total death \% for all positive cases, broken down by vaccine type (deaths / positive cases) over the time period of December 2020 - July 2021. Vaccinated cases tested positive 14 days after their final dose. 
Comparison (events per 100,000 persons per week) between Pfizer-BioNTech with Sinopharm and Unvaccinated with Sinopharm.

(a)

\begin{tabular}{|c|c|c|c|c|c|c|c|c|c|c|c|c|}
\hline & \multicolumn{12}{|c|}{ Pfizer-BioNTech vs Sinopharm } \\
\hline & \multicolumn{4}{|l|}{ ALL } & \multicolumn{4}{|l|}{ Over 50} & \multicolumn{4}{|l|}{ Under 50} \\
\hline & $\begin{array}{l}\text { Rate } \\
\text { (Pfizer) }\end{array}$ & $\begin{array}{l}\text { Rate } \\
\text { (Sino) }\end{array}$ & $\mathrm{p}$ & OR & $\begin{array}{l}\text { Rate } \\
\text { (Pfizer) }\end{array}$ & $\begin{array}{l}\text { Rate } \\
\text { (Sino) }\end{array}$ & $\mathrm{p}$ & OR & $\begin{array}{l}\text { Rate } \\
\text { (Pfizer) }\end{array}$ & $\begin{array}{l}\text { Rate } \\
\text { (Sino) }\end{array}$ & $\mathrm{p}$ & OR \\
\hline Infections & 136.28 & 350.53 & $<.001$ & 0.44 & 128.44 & 366.57 & $<.001$ & 0.39 & 139.65 & 345.52 & $<.001$ & 0.46 \\
\hline Hospitalizations & 2.82 & 28.36 & $<.001$ & 0.13 & 6.59 & 62.11 & $<.001$ & 0.12 & 1.74 & 14.74 & $<.001$ & 0.17 \\
\hline ICU admissions & 0.08 & 2.29 & $<.001$ & 0.04 & 0.36 & 6.62 & $<.001$ & 0.06 & 0.0 & 0.49 & $<.05$ & 0.00 \\
\hline Deaths & 0.22 & 1.64 & $<.001$ & 0.15 & 0.96 & 5.45 & $<.001$ & 0.18 & 0.0 & 0.16 & NS & 0.00 \\
\hline
\end{tabular}

(b)

\begin{tabular}{|c|c|c|c|c|c|c|c|c|c|c|c|c|}
\hline & \multicolumn{12}{|c|}{ Unvaccinated vs Sinopharm } \\
\hline & \multicolumn{4}{|l|}{ ALL } & \multicolumn{4}{|l|}{ Over 50} & \multicolumn{4}{|l|}{ Under 50} \\
\hline & $\begin{array}{l}\text { Rate } \\
\text { (Unvax) }\end{array}$ & $\begin{array}{l}\text { Rate } \\
\text { (Sino) }\end{array}$ & $\mathrm{p}$ & OR & $\begin{array}{l}\text { Rate } \\
\text { (Unvax) }\end{array}$ & $\begin{array}{l}\text { Rate } \\
\text { (Sino) }\end{array}$ & $\mathrm{p}$ & OR & $\begin{array}{l}\text { Rate } \\
\text { (Unvax) }\end{array}$ & $\begin{array}{l}\text { Rate } \\
\text { (Sino) }\end{array}$ & $\mathrm{p}$ & OR \\
\hline Infections & 642.96 & 350.53 & $<.001$ & 1.72 & 781.32 & 366.57 & $<.001$ & 2.02 & 631.66 & 345.52 & $<.001$ & 1.70 \\
\hline Hospitalizations & 51.06 & 28.36 & $<<.001$ & 1.78 & 225.55 & 62.11 & $<.001$ & 3.49 & 33.98 & 14.74 & $<.001$ & 2.35 \\
\hline ICU admissions & 6.39 & 2.29 & $<.001$ & 2.70 & 41.44 & 6.62 & $<.001$ & 5.92 & 2.65 & 0.49 & $<.05$ & 5.09 \\
\hline Deaths & 4.42 & 1.64 & $<.001$ & 2.72 & 37.17 & 5.45 & $<.001$ & 6.56 & 1.35 & 0.16 & NS & 7.65 \\
\hline
\end{tabular}

Table 4: Pairwise comparison (events per 100,000 persons per week) of the Pfizer/BioNtech and the Sinopharm vaccines (a) and Unvaccinated versus Sinopharm vaccinated individuals (b) in age cohorts $>50$ and $<50$ showing the rates of infections, hospitalizations, ICU admissions and deaths, $\mathrm{p}$ values and odds ratios. All rates are events per 100,000 of the sample population. Note that the $\mathrm{p}$ values for the comparison were all $<0.01$, except ICU admissions in "under 50" cohort and deaths for the Sinopharm versus Pfizer comparison and Sinopharm versus Unvaccinated comparison (in bold). 
Infected Rate per Vaccine (all ages)

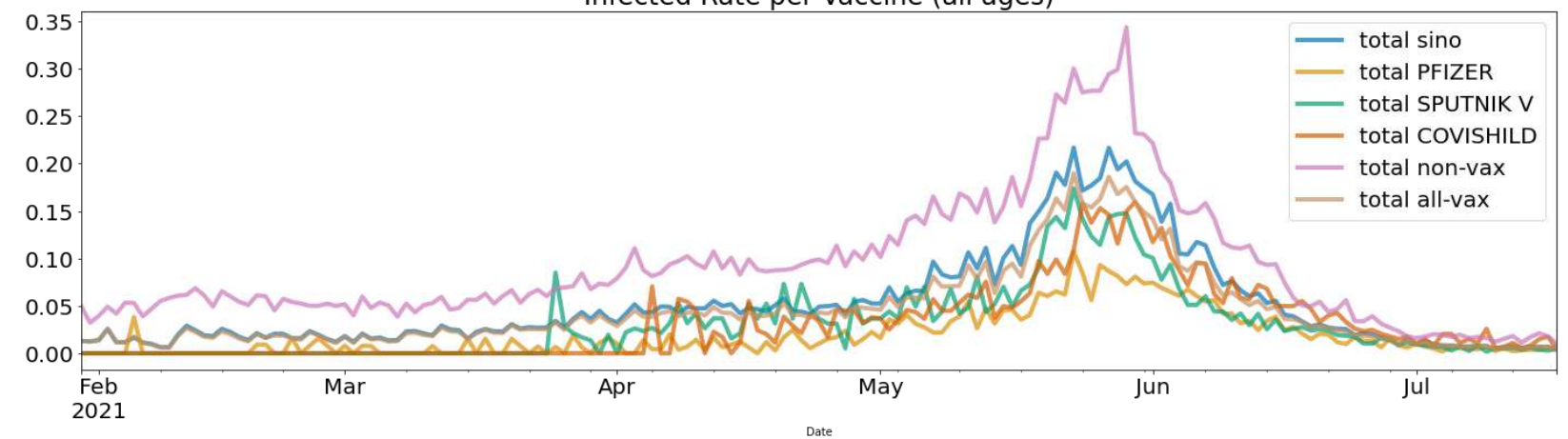

Hospitalised Rate per Vaccine (all ages)

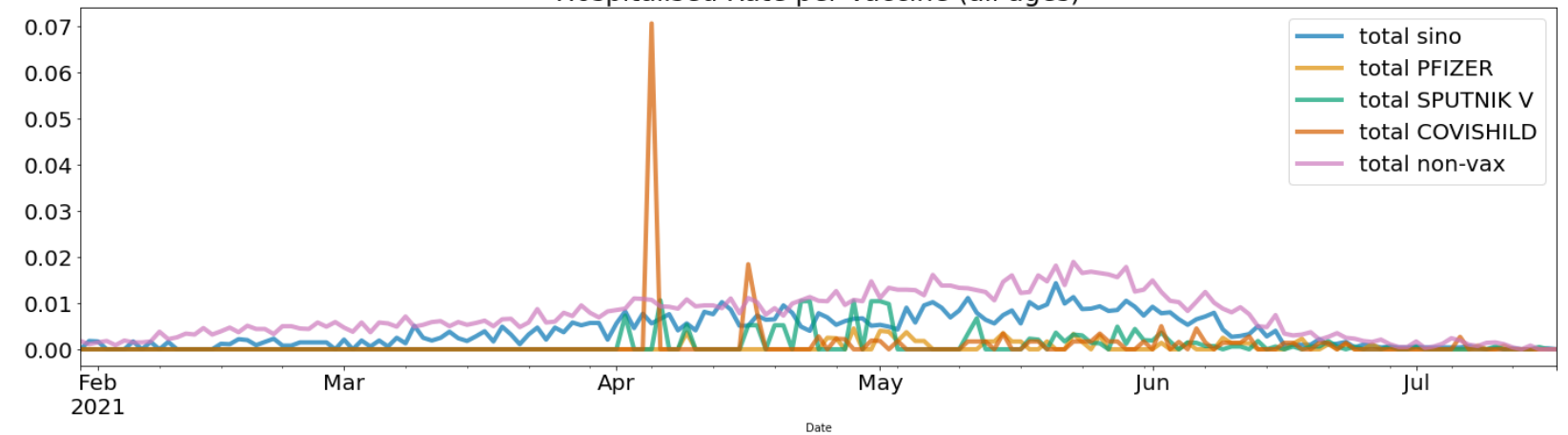

Icu Rate per Vaccine (all ages)

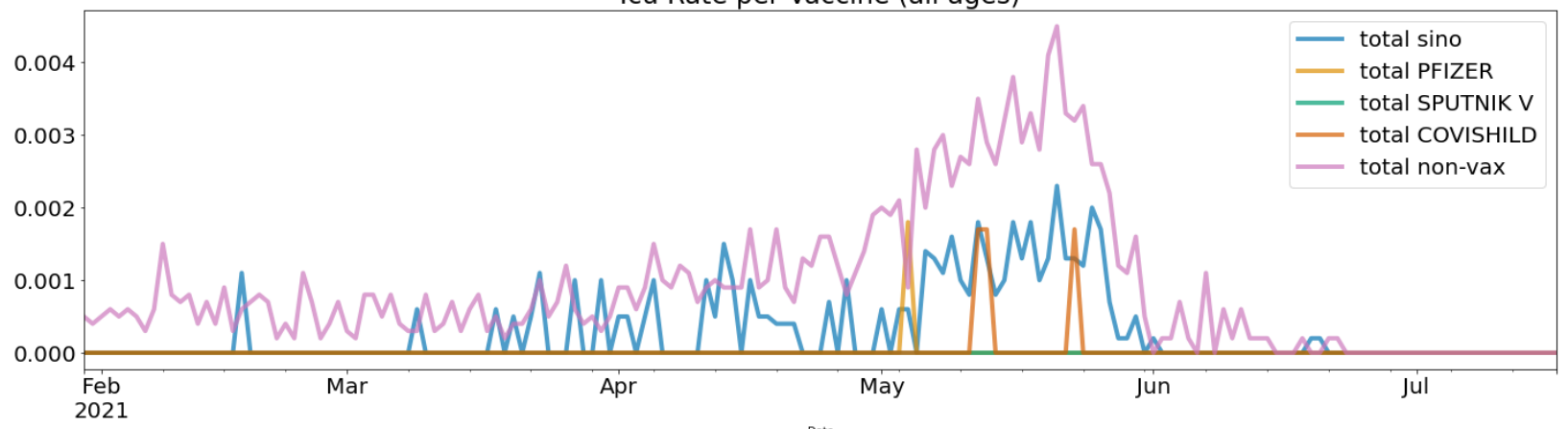

Deaths Rate per Vaccine (all ages)

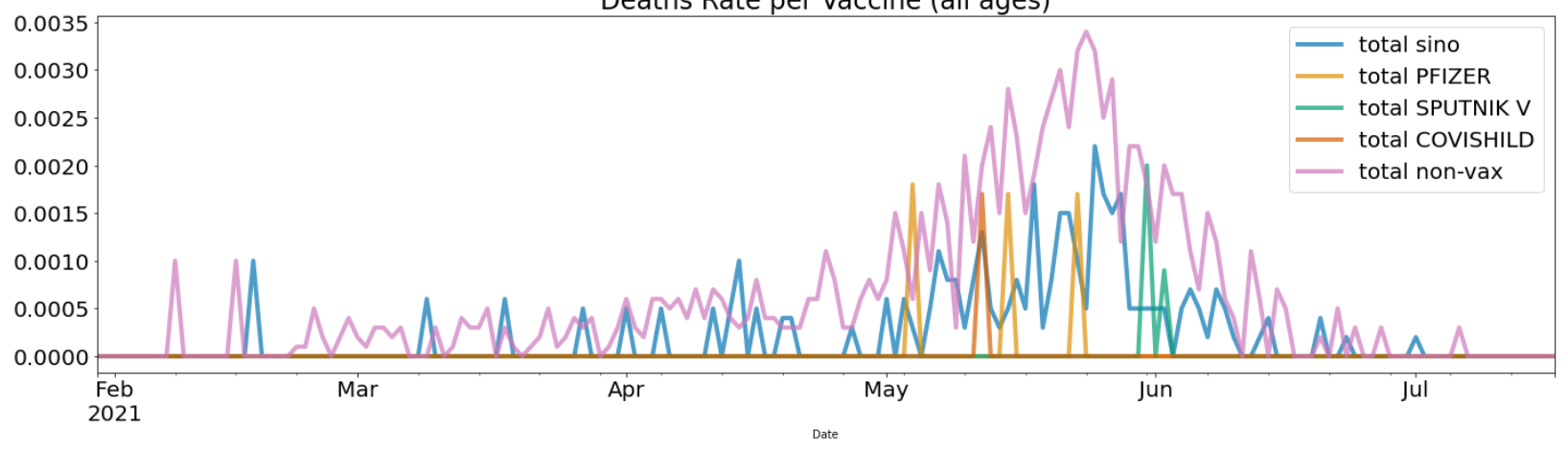

Figure 3: Timeline (events per person at risk per day) of infection, hospitalizations, ICU admissions and deaths for four vaccines compared to unvaccinated controls between Jan 30, 2021 to July 17th 2021. 

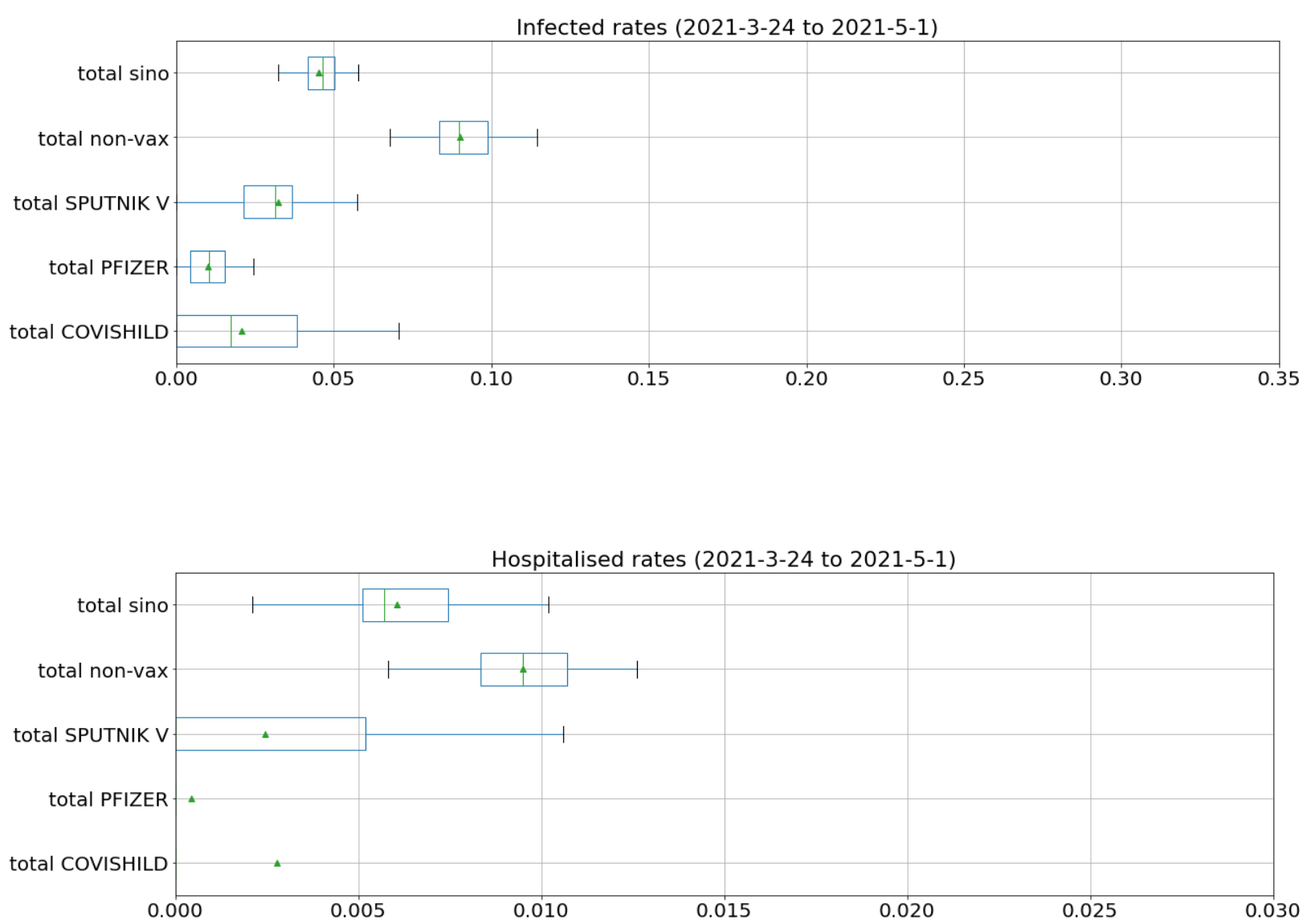

(a)

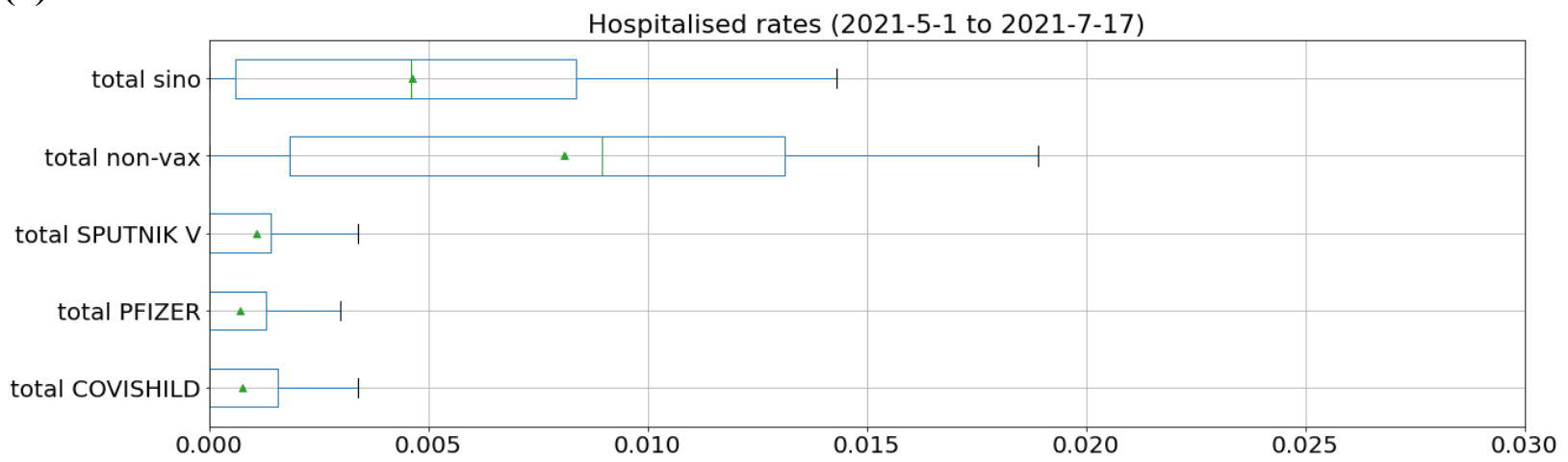

(b)

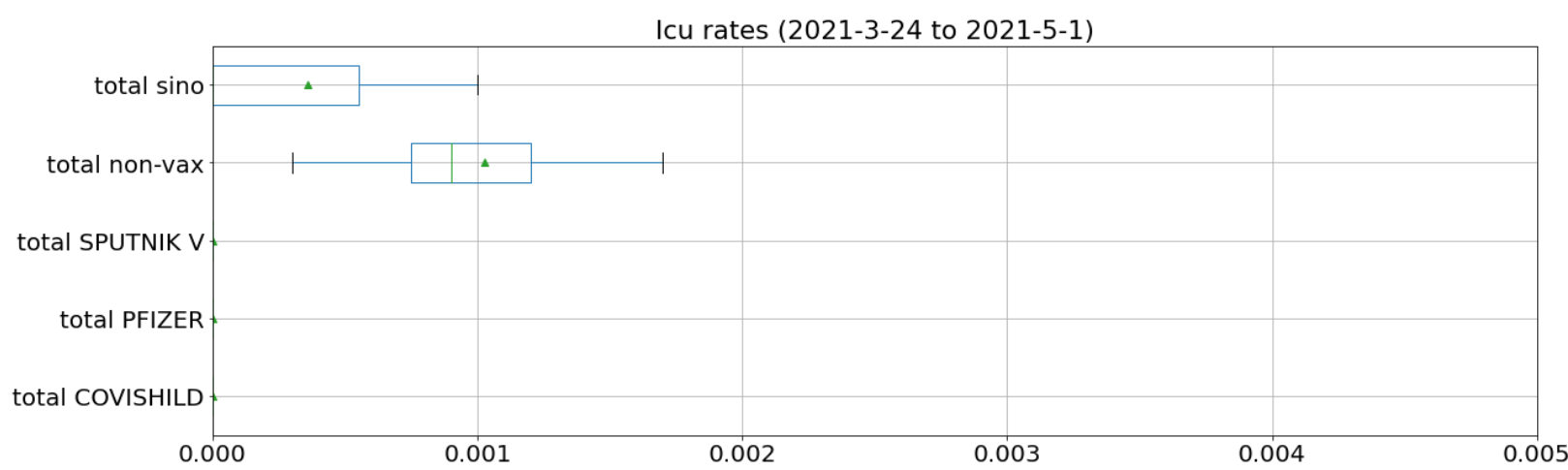


(c)

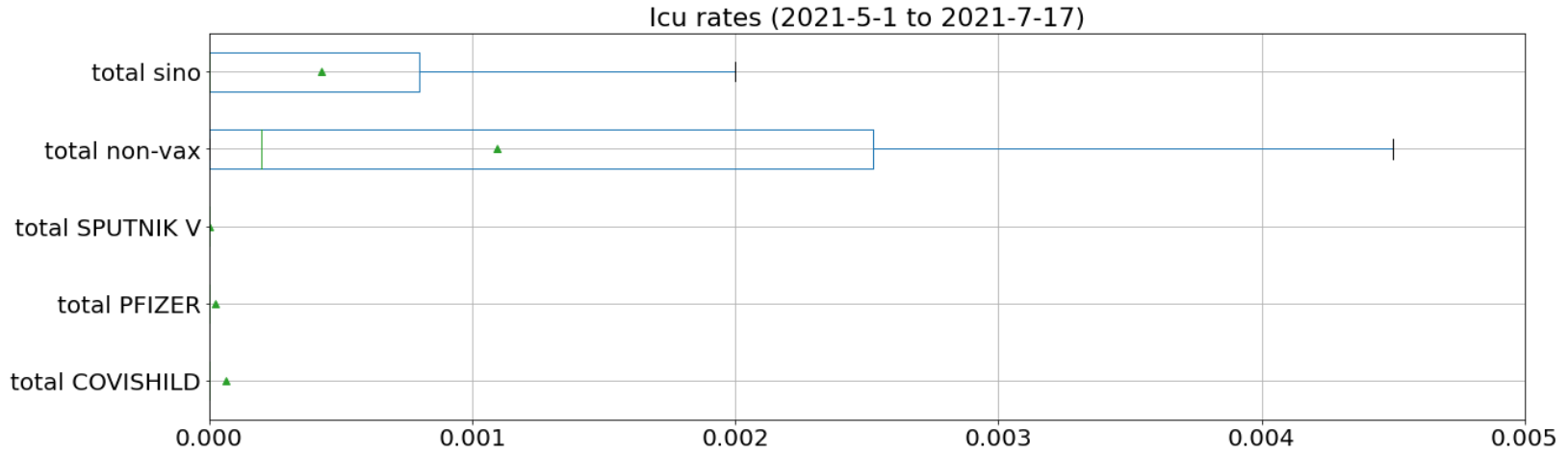

(d)

Deaths rates (2021-3-24 to 2021-5-1)

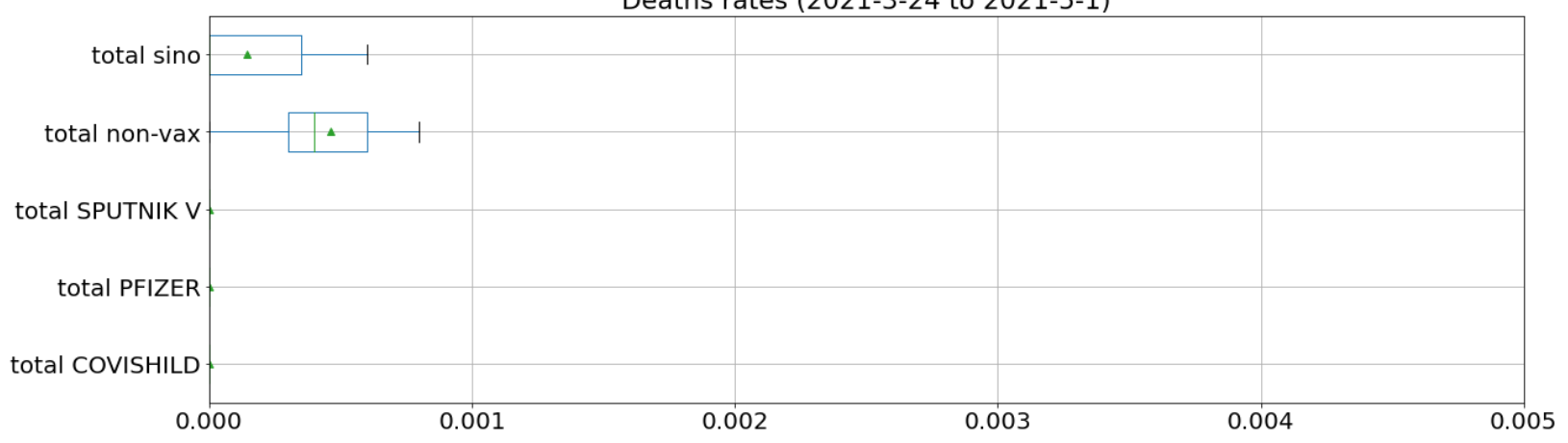

(e)

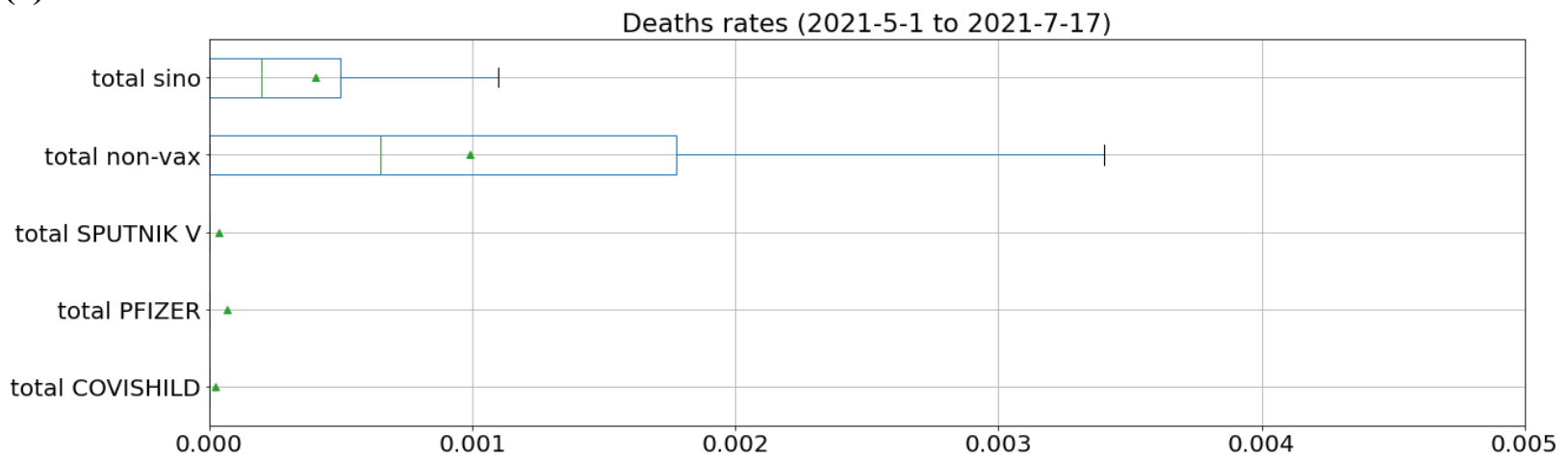

(f) 

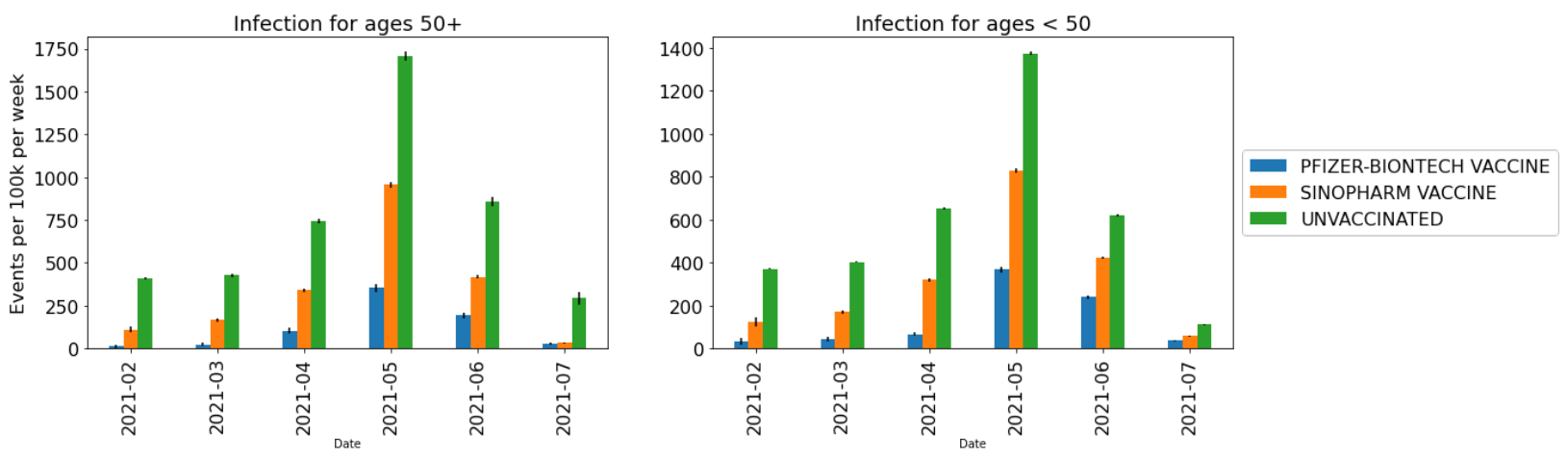

(g)
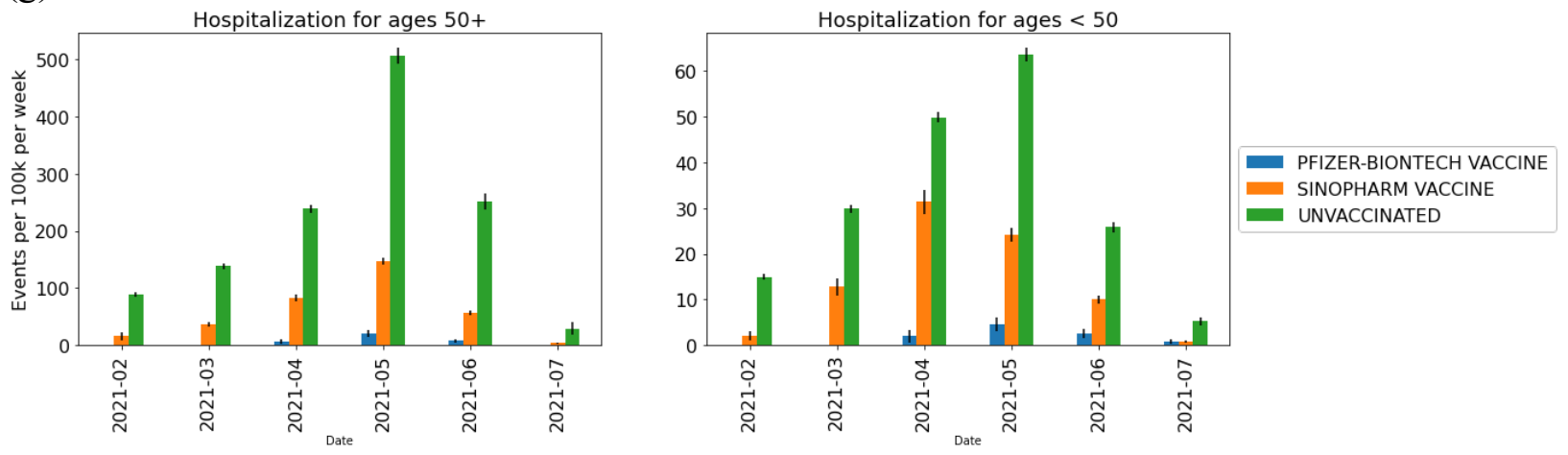

(h)
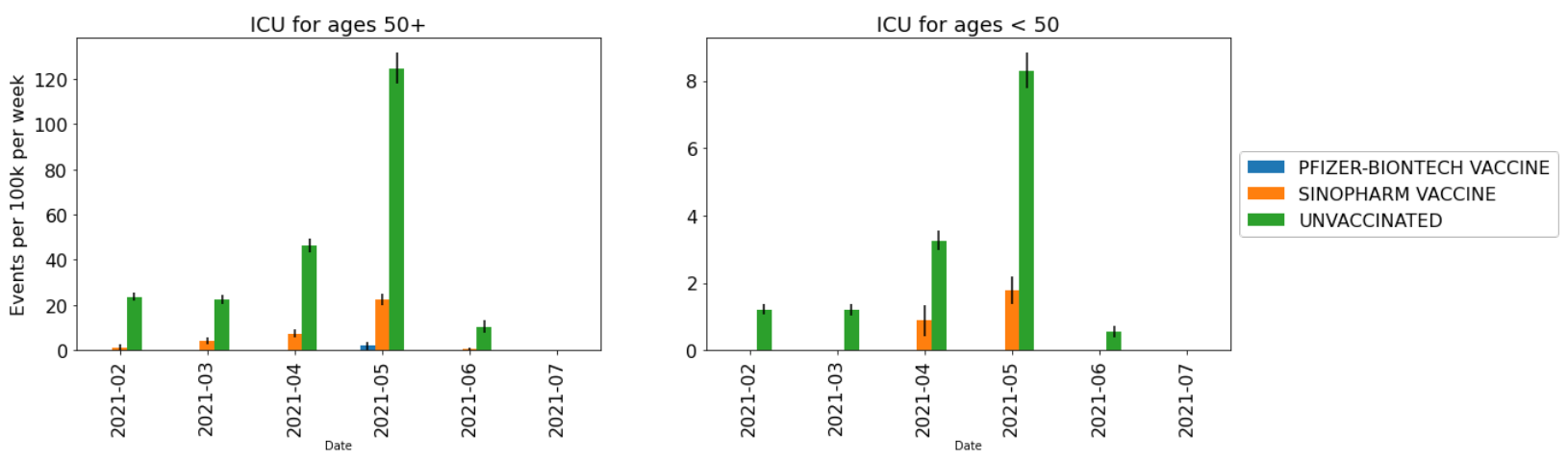

(i) 

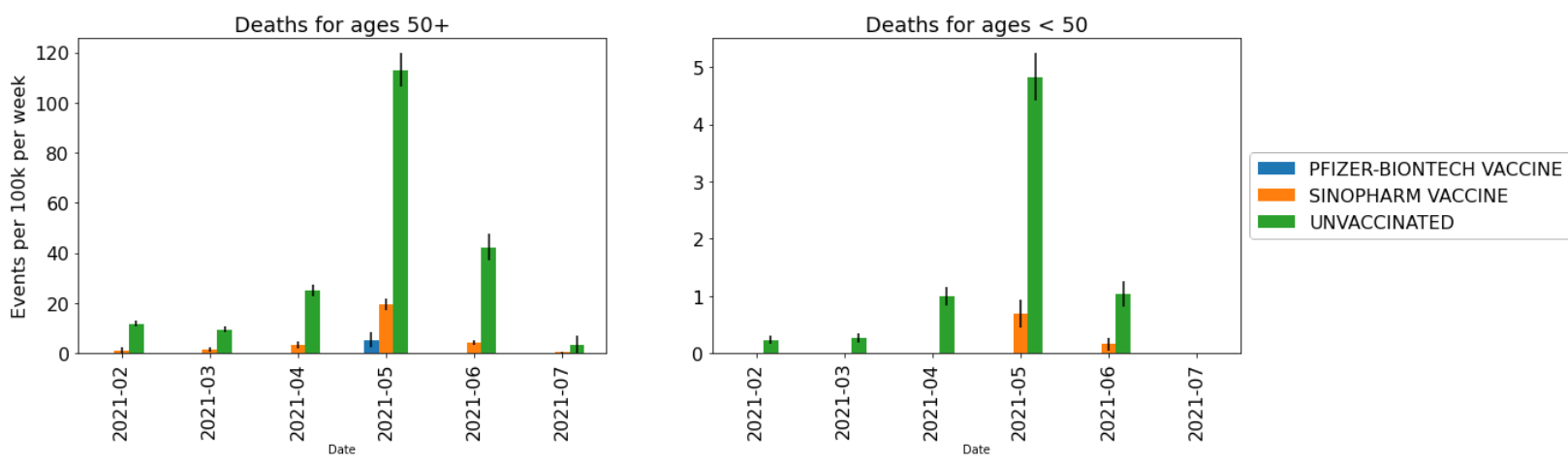

(j)

Figure 4: (a-f) Box plots illustrating the distribution of rates for infections, hospitalizations, ICU admissions, and deaths. Where there is sufficient data, the whiskers illustrate the maximum and minimum, and boxes encompass the first and third quartiles. Median is displayed as a vertical line in the box. Data are split before and after May 1, 2021 when the Delta variant became prevalent in the studied population. (g-j) bar plots representing the events per 100,000 vaccine recipients from February 2021 to July 2021, comparing infections (g), hospitalizations (h), ICU admissions (i) and deaths (j). For (g) to (j), breakthrough infections after the Sinopharm and Pfizer vaccine are compared against unvaccinated individuals, divided by age cohorts $>50$ years (left plot) and $<50$ years (right plot). Standard error is shown as whiskers for each of these plots $(g-j)$.
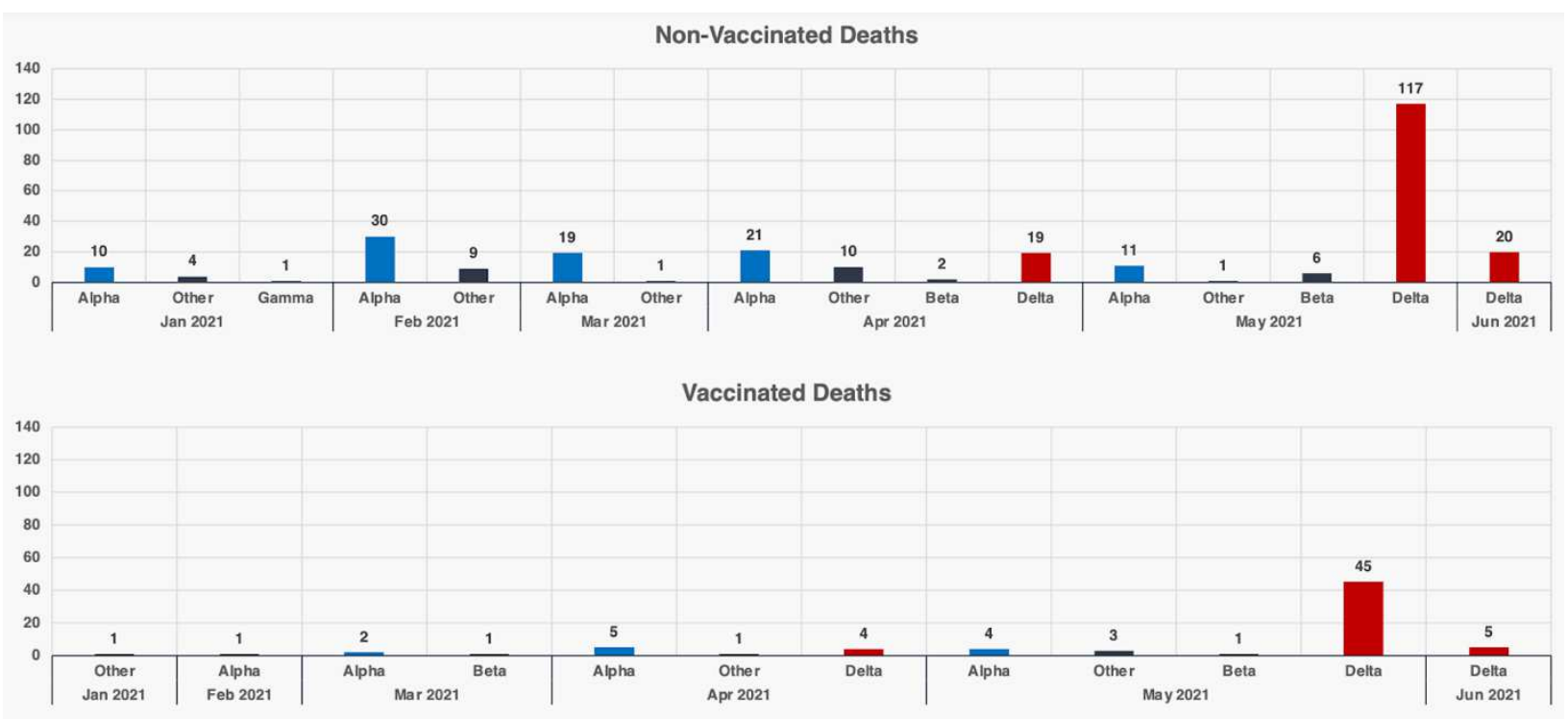

Figure 5: Variant sequencing for SARS-Cov2 infected individuals who died (presumptively from SARSCov2 infection). Variants detected between January 12021 to June 302021 are shown in the following 
brackets: Alpha (B1.1.7), Beta (1.351), Gamma (B.1.28.1) and Delta ( B.1.617.1 and B.1.617.2) and Other for vaccinated and unvaccinated individuals.

\section{METHODS:}

\section{Vaccination program in Bahrain:}

The Kingdom of Bahrain has introduced a series of measures to ensure equal access to the vaccines regardless of race, age, nationality, gender, or income. Vaccines were administered at no cost. Thirtyone vaccination sites were established in the country to ensure access. Protocols were also available for registration, counselling/consultation vaccination and observation. The sites were geographically distributed at 27 primary care centers, The International Exhibition and Convention Centre, Sitra Mall, King Hamad University Hospital and Bahrain Defense Force Hospital mobile vaccination units were utilized to provide the vaccine to senior citizens, persons with special needs, inmates, and others who are unable to receive the vaccine at one of the designated vaccination centers.

The national vaccination for the general population started on December 16th 2020 through 31 vaccination centers. Initially, high risk populations (i.e., frontline workers, individuals $>60$ years of age, and patients with morbid obesity, any immunocompromised state, diabetes, cardiopulmonary disease and hypertension) were given priority when booking appointments over the general population. By December 16, 2020, vaccination was extended to the general population. Four COVID-19 vaccines were used. The Sinopharm vaccine was deployed first, in part because Bahrain was involved in clinical trials with Sinopharm, followed by Pfizer/BioNtech, Sputnik V and AZ-Covishield. Note that the early cohort of Sinopharm vaccinees have been censored.

\section{Data collection database:}

A country specific Information system (ISEHA) has all medical history of individuals and is centralised and used for all Covid related health information. Anonymized electronic health records were retrieved for the study period December 9th 2020 - July 17 2021.These records include: (a) Patient demographics, a random ID used to link records, year of birth, sex, coded geographical location of residence, nationality and chronic comorbidities including: cardiovascular disease, type 2 diabetes, high blood pressure, immunosuppression, high BMI, chronic kidney disease (CKD) and chronic obstructive pulmonary disease (COPD).

\section{Identification of Positive cases and variants:}

All cases were diagnosed based on RT-PCR tests of nasopharyngeal samples. The majority of RT-PCR tests were conducted using Thermo Fisher Scientific (Waltham, MA) TaqPath 1-Step RT-qPCR Master Mix, CG (catalog number A15299) on the Applied Biosystems (Foster City, CA) 7500 Fast Dx RealTime PCR Instrument. The assay used followed the World Health Organization protocol and targeted the E gene. ${ }^{9}$ If positive, the sample was confirmed by RdRP and $\mathrm{N}$ genes. The $\mathrm{E}$ gene $\mathrm{CT}$ value was reported and used in this study. CT Values $<37$ were considered positive. Whole genome sequencing was used to identify the common variants of concerns using illumina/ARTIC and COVIDSeq protocols. The data were analysed with the Abiomix platform. Sequencing was undertaken at the 
national COVID-19 Molecular public health laboratory where all the samples get tested there. Spike gene target status on PCR was used as a second approach for identifying each variant.

\section{Statistical analysis:}

Statistical analysis was performed as follows. Analysis was performed using statsmodels 0.10.2. Positive events were filtered to be either from persons of 18 years old or older and to unvaccinated individuals (once vaccinated count as this) or from individuals who were fully vaccinated on or after 2021-01-30. Counts of who is at risk in each subgroup, for the purpose of rate denominators, reflect available data (unvaccinated) or all individuals (living or dead) who were fully vaccinated after this date.

Odds ratio analysis. Event rate comparisons were made in terms of odds ratios (for hospitalization, ICU, death) using Cochran Mantel Haenszel analysis of event counts stratified by day. The first analyses were made using the full study period comparing Pfizer to Sinopharm and unvaccinated to Sinopharm. Similar analyses were made when appropriately small p-values were found in the first analysis for the data broken by month or broken by before/after 2021-05-01.

Event rate displays. To display event rates the computation for a given time period went as follows: 1) Compute daily rates (i.e. event count in a given day/ at risk count for that day). 2) Sum the daily rates over the time period. 3) Convert this figure to the units of per $100 \mathrm{~K}$ persons and per 7 days, taking account of the number of days in the time period. Similar calculations were made for the variance of this quantity using the delta method and assuming that the count variance on each day is an independent Poisson (given the true rate parameter), that is plugging in the observed count as a variance for the count and propagating this to compute a variance for the computed rate. This was square-rooted to give a standard error for the plots.

PCR Positive event proportion analysis To give confidence intervals to the proportions of events, Jeffreys intervals were used using an alpha value of 0.01 . In this way, when considering one event outcome at a time, the five vaccine conditions can be considered to have simultaneous confidence intervals at 0.05 -level (i.e. the FWER is controlled). For formal pairwise comparisons between vaccination conditions of such a rate, the five all-pairs two-by-two tables of event/non-event vs vaccinegroup1/vaccine-group2 were analyzed using Fisher exact p-values. The resulting 10 p-values were converted to corrected p-values using the Holm-Sidak method.

We wish to thank Anupam Pathakfor analyzing and generating the Figures from the raw data, Ahmad Humaidin for assembling the raw data, Jake Garrison for generating the time series plots and the Kingdom of Bahrain for providing anonymized raw data for the project.

\section{References:}

1. Polack, F. P. et al. Safety and Efficacy of the BNT162b2 mRNA Covid-19 Vaccine. N. Engl. J. Med.

383, 2603-2615 (2020).

2. Baden, L. R. et al. Efficacy and Safety of the mRNA-1273 SARS-CoV-2 Vaccine. N. Engl. J. Med. 384, 403-416 (2021). 
3. Voysey, M. et al. Safety and efficacy of the ChAdOx1 nCoV-19 vaccine (AZD1222) against SARS-

CoV-2: an interim analysis of four randomised controlled trials in Brazil, South Africa, and the UK. The Lancet 397, 99-111 (2021).

4. Al Kaabi N, et al. Effect of 2 Inactivated SARS-CoV-2 Vaccines on Symptomatic COVID-19 Infection in Adults: A Randomized Clinical Trial. JAMA 326, 35-45 (2021).

5. Logunov DY, et al. Safety and efficacy of an rAd26 and rAd5 vector-based heterologous prime-boost COVID-19 vaccine: an interim analysis of a randomised controlled phase 3 trial in Russia. The Lancet 397, 671-681 (2021).

6. Lopez Bernal, J. et al. Effectiveness of Covid-19 Vaccines against the B.1.617.2 (Delta) Variant. $N$. Engl. J. Med. 385, 585-594 (2021).

7. Stokes EK, Zambrano LD, Anderson KN, et al. Coronavirus disease 2019 case surveillance — United States, January 22-May 30, MMWR Morb Mortal Wkly Rep 2020;69:759-765 (2021)

8. O’Driscoll, M. et al. Age-specific mortality and immunity patterns of SARS-CoV-2. Nature 590, 140145 (2021).

9. Corman VM, Landt O, Kaiser M, et al. Detection of 2019 novel coronavirus (2019-nCoV) by real-time RT-PCR. Euro Surveill 25 (2020).

\section{Supplementary Figures:}

Outcomes Rates among vaccinated and unvaccinated individuals by age cohorts $>\mathbf{5 0}$ and $\leq \mathbf{5 0}$ years

\section{a. Infection}


Infected Rate per Vaccine (above 50 yo)

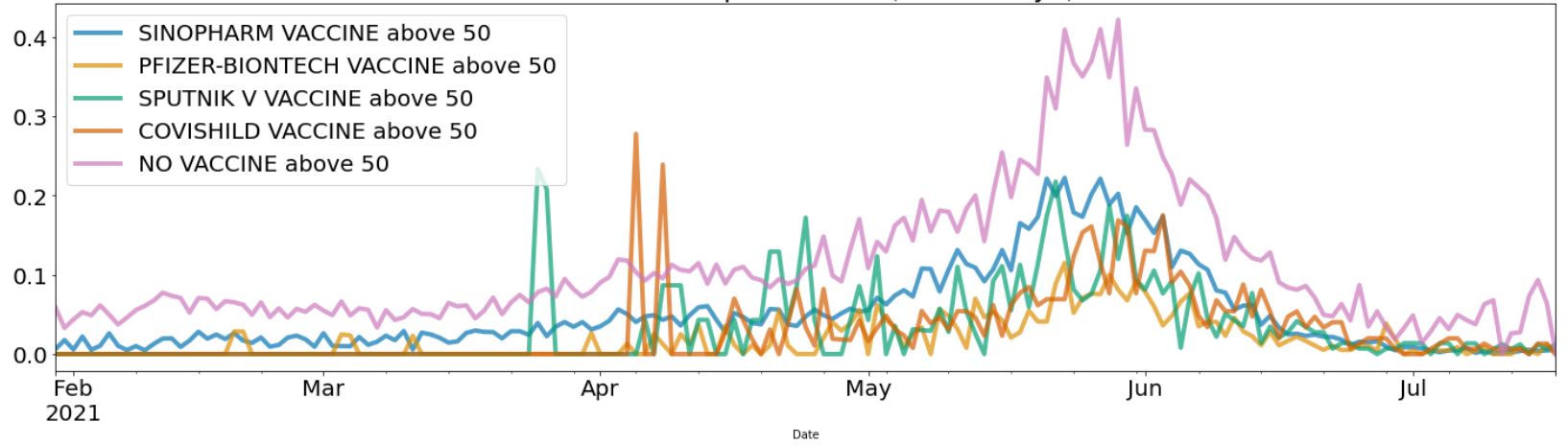

Infected Rate per Vaccine (below 50 yo)

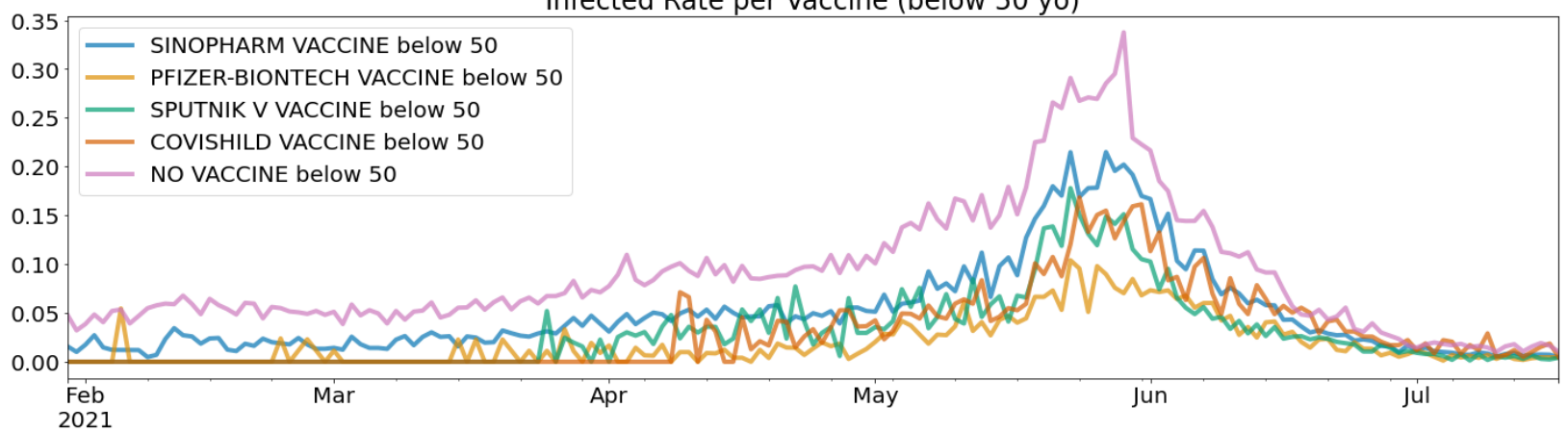

Date

\section{b. Hospitalization}

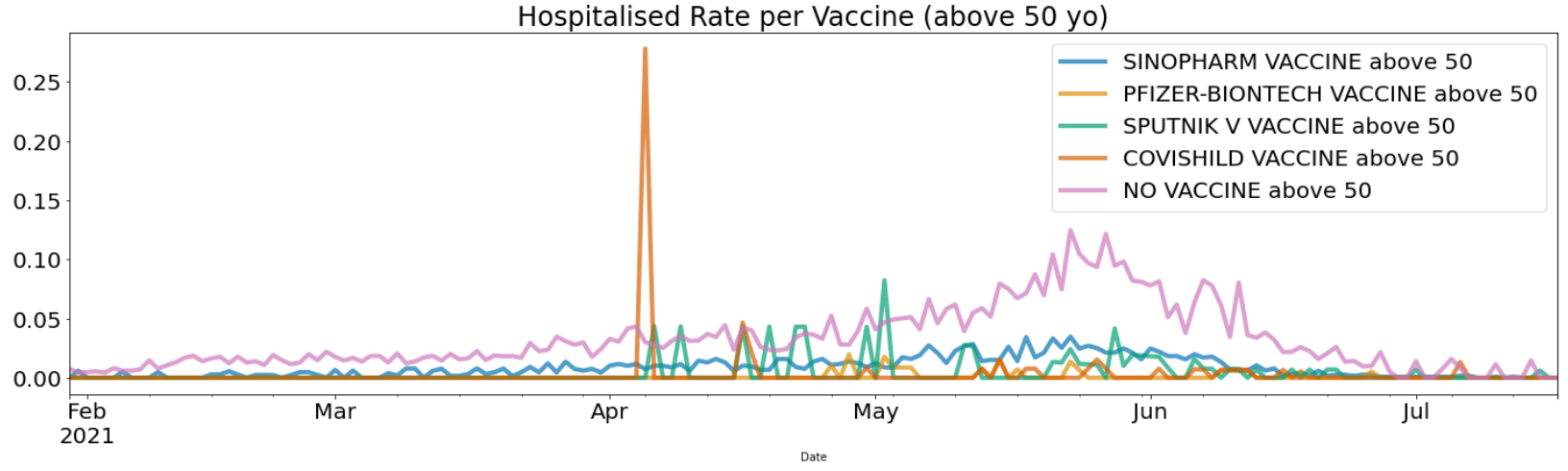

Hospitalised Rate per Vaccine (below 50 yo)

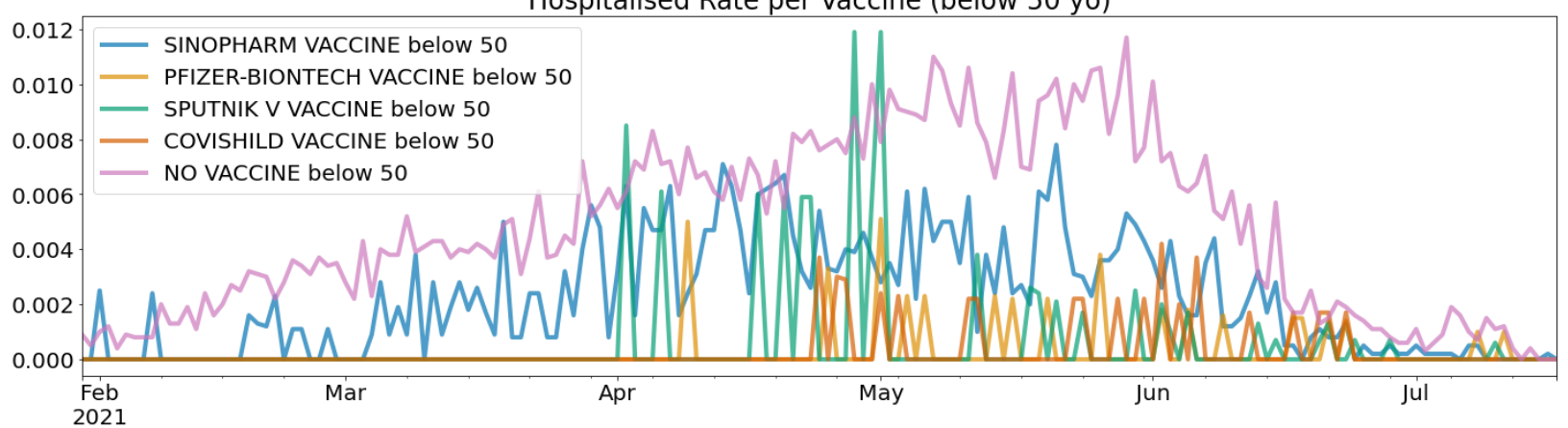




\section{(c) ICU Admissions}

Icu Rate per Vaccine (above 50 yo)

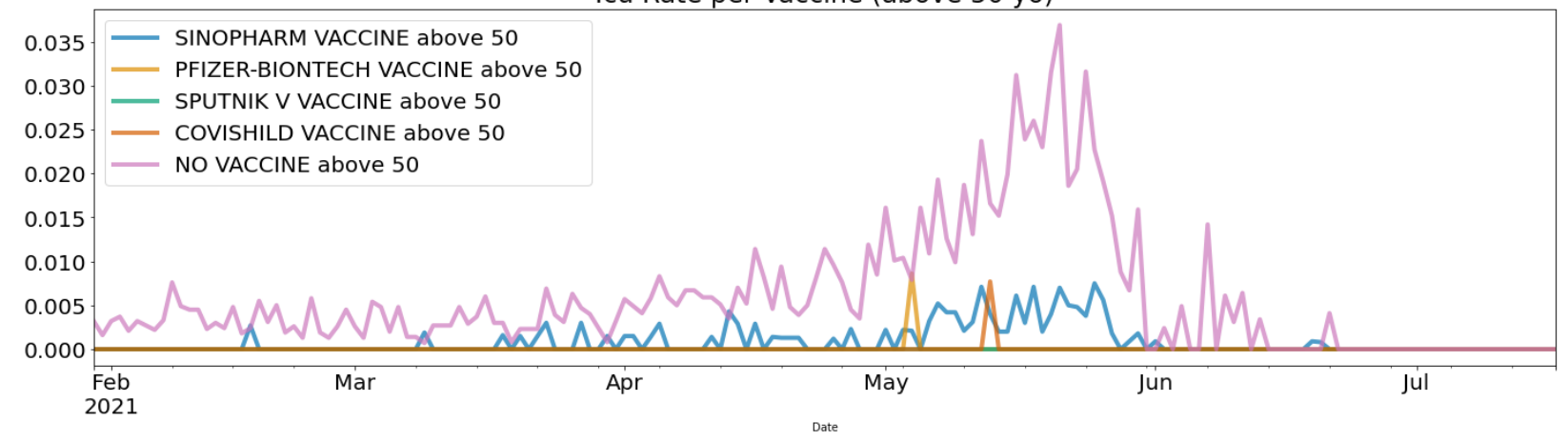

Icu Rate per Vaccine (below 50 yo)

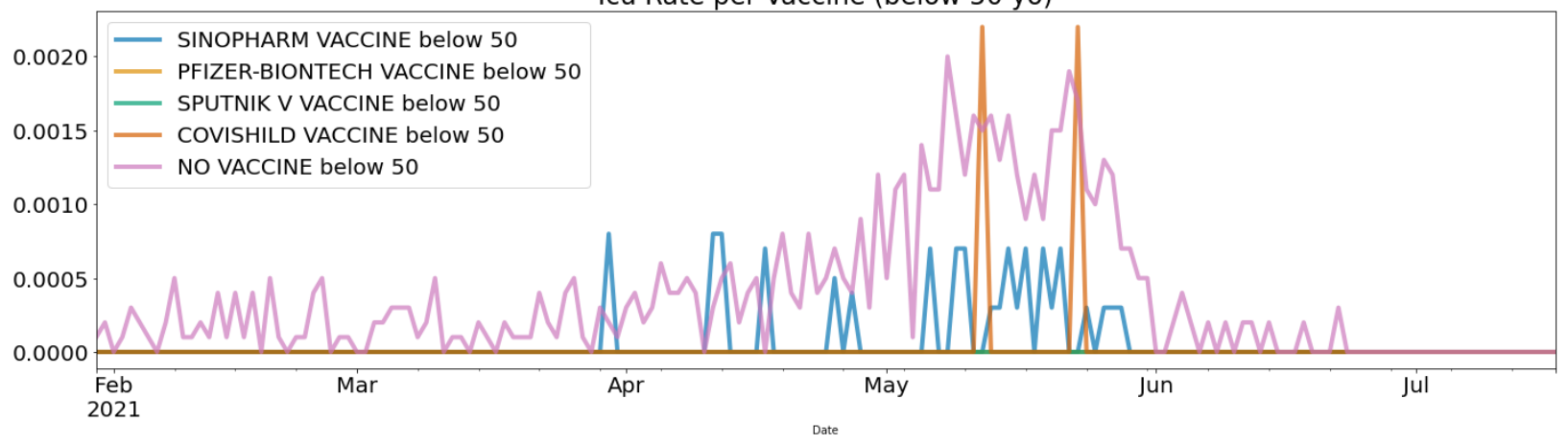

\section{(d) Deaths}

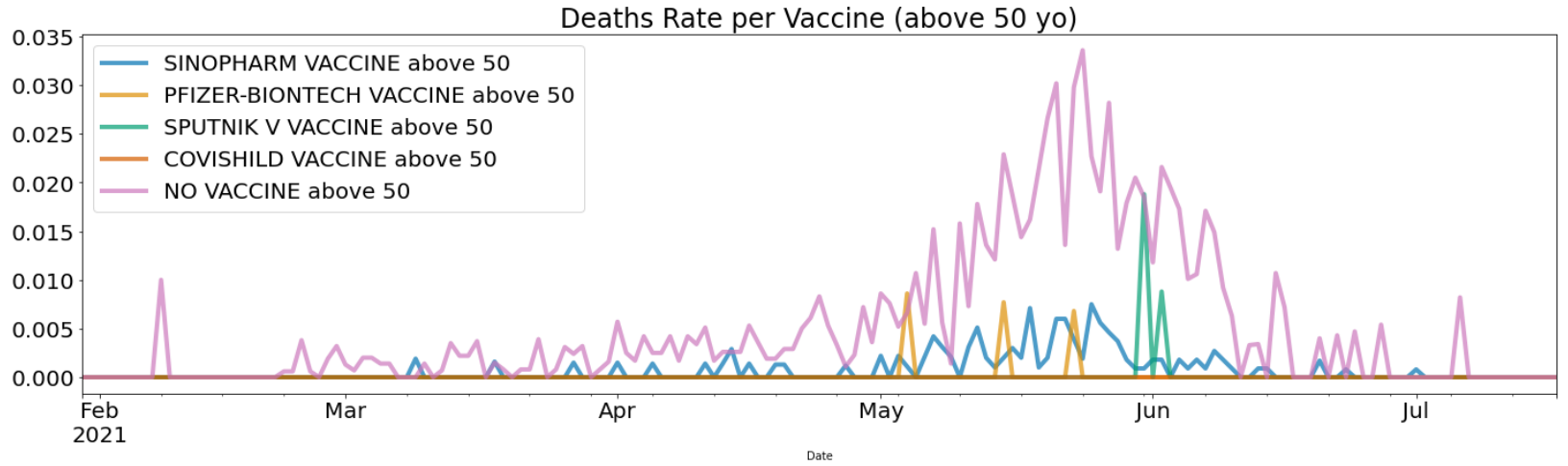


Deaths Rate per Vaccine (below 50 yo)

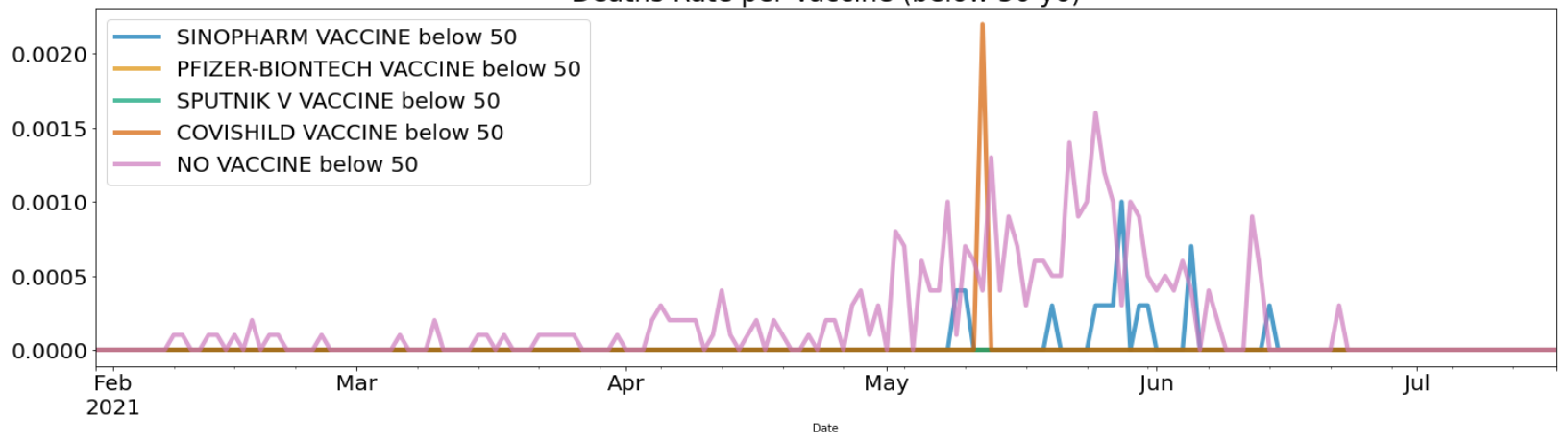

Figure S1: Time series illustrating rates of a) Infections, b) Hospitalizations, c) ICU admissions, and d) deaths for all vaccines and unvaccinated for the above and below 50 age cohort. Events per 100k population per week are also shown as a time series where a maximum is seen in the month of May (when Delta became the dominant variant). 